\title{
Photocatalytic Degradation of Organic Pollutant using Reduced Graphene Oxide
}

\author{
Raja. A, Selvakumar. K, Asath Bahadur. S, Swaminathan. M
}

\begin{abstract}
A simple eco friendly preparation of reduced graphene oxide from graphene oxide using strawberry extract is reported. As prepared reduced graphene oxide were characterized by X-Ray Diffraction, $U V$-Vis spectroscopy, Scaning electron microscopy and degradation performane of $M B$. The reduced graphene oxide was effectively degradation of $M B$.

Keywords-Methyle Blue; Degradation;
\end{abstract}

\section{INTRODUCTION}

The Polluting substance of textile dye waste particularly MB in ground water its environmental pollution and health issues. MB is mostly used as an textile industries, the dyes to mixed in river and soil to destroy water and soil pollution to induced health issues. The MB has damage the ecological system, the dyes cannot be remove in soil and water. It is found in surface water and underground water all over the world. A small amount of $\mathrm{MB}$ in water to induced health issues and environmental pollution. It is big task for for MB removal in ecosystem [1]. Types of methods to preparation of graphene from graphene oxide like chemical method and green route [2-4]. In this preparation method to involved by anthocyanins act as a reducing agent, stabilizer, capping agent to control the crystallization [5-8]. Nowadays reduced graphene oxide has create more application due to tunable band gap, large surface area, easy processibility, compatibility with different substrate. It has been very suitable material for the charge traping graphene sheet for memory devices. The oxidized graphene oxide act as an insulating material and partially oxidized graphene oxide act as a semiconducting material. As prepared reduced graphene oxide such as dispersible in water, adjustable band gap biocompatibility and good photocatalytic material. In the present work, the photocatalytic decomposition of reduced graphene oxide nanosheet by photodecomposition of $\mathrm{MB}$ under UV light irradiation.

Graphite flakes were purchased from alfa aesar, India. Sulphuric acid, hydrochroloric acid and hydrogen peroxide were purchased from sigma aldrich. UV-visible spectra were

Revised Manuscript Received on December 05, 2019.

* Correspondence Author

A. Raja, Multifunctional Materials Research Laboratory, Department of Physics, Kalasalingam University, Krishnankoil, Tamil Nadu, India, Email:rajaannamalai88@gmail.com

K. Selvakumar, Nanomaterials Laboratory, Department of Chemistry, International Research Centre, Kalasalingam Academy of Research and Education (Deemed to be University), Krishnankoil - 626126, India. Email: selvachemistk@gmail.com

S. Asath Bahadur*, Multifunctional Materials Research Laboratory, Department of Physics, Kalasalingam University, Krishnankoil, Tamil Nadu, India, Email: s_a_bahadur@yahoo.co.in

M, Swaminathan, Nanomaterials Laboratory, Department of Chemistry, International Research Centre, Kalasalingam Academy of Research and Education (Deemed to be University), Krishnankoil - 626126, India. Email: m.swaminathan@klu.ac.in

\section{EXPERIMENTAL DETAILS}

recorded with Hitachi (U-2001, TOKYO) and Fourier transform infrared analysis were recorded using Nicolet (Madison). The X-ray diffraction spectrum was obtained by Rigaku X-ray diffractometer (miniflex), the range of $2 \theta=2-700$. Transmission electron microscopy (TEM) images were recorded at JEOL 2100k microscope, the operating voltage at $200 \mathrm{kv}$, was used to get Transmission electron microscopy images.

\section{MATERIALS AND METHODS}

\section{A. Materials and characterization}

Silver nitrate, Neodymium acetate, Zinc acetate raw materials are purchased from Sigma Aldrich. And Oxalic acid, Sodium hydroxide, $\mathrm{C}_{4} \mathrm{H}_{6} \mathrm{O}_{4} \mathrm{Zn} \cdot 2 \mathrm{H}_{2} \mathrm{O}$, Methylene blue (Merck Chemicals, India) are the other raw materials. All the solutions are prepared to use distilled water. The Scanning electron microscope with a model (EVO-80, CARL ZEISS) and the (EDX) spectroscopy (AMETEK-EDAX (Z2e Analyzer)) is used to predict surface morphology and the presence of elements to the nanocomposite. The crystalline structure of the sample was analyzed by powder X-ray diffraction (PXRD) (Shimadzu XRD-6000 X-ray diffractometer $(\mathrm{Cu} \mathrm{K \alpha}$ source)). The bandgap was analyzed by UV-Vis diffuse reflection spectroscopy (DRS) was performed on a Shimadzu UV-2450 spectrophotometer. The UV-visible spectroscopy with the model Shimadzu UV-1800 spectrophotometer is used to analyze the degradation efficiency of the collecting photocatalytic degradation samples.

\section{A. Synthesis of graphene oxide:}

Preparation of graphene oxide by modified hummers method. $6 \mathrm{~g}$ of natural graphite powder was mixed $\mathrm{H}_{2} \mathrm{SO}_{4}$ with $1.5 \mathrm{~g}$ of sodium nitrate in $230 \mathrm{~mL}$ of $\mathrm{H}_{2} \mathrm{SO}_{4}$ in an ice bath to cooled 0 ${ }^{0} \mathrm{C} .18 \mathrm{~g}$ of potassium permanganate was added very slowly to the mixture under stirring and maintaining the temperature below $5{ }^{\circ} \mathrm{C}$. In this light green colour suspension was stirred for $24 \mathrm{~h}$ under maintain at $10{ }^{\circ} \mathrm{C}$ using ice bath. Then the mixture suspension diluted with $300 \mathrm{~mL}$ of double distilled water under stirring for $1.5 \mathrm{~h}$ and the temperature was maintained at $98{ }^{\circ} \mathrm{C}$. The suspension was diluted by 1 litre distilled water and $30 \% \mathrm{H}_{2} \mathrm{O}_{2}$ was added to continuous stirring for $24 \mathrm{~h}$ under ice bath. The suspension color was changed into brown. After, the suspension was centrifuged and washed with $1 \mathrm{M} \mathrm{HCl}$ solution and double distilled water. The final product was dispersed in double distilled water and ultrasonic treatment for $2 \mathrm{~h}$. Then the final product was filtered and washed with ethanol, dried under vacuum at $10^{\circ}$ C for 24 hrs [9].

\section{Published By:}




\section{Synthesis of reduced graphene oxide}

$30 \mathrm{mg}$ strawberry were crushed and change into $100 \mathrm{~mL}$ beaker with water. As prepared extract was heated at $100{ }^{\circ} \mathrm{C}$ for $10 \mathrm{~min}$. after the mixture extract solution was allowed to cool down to atmospheric temperature and filtered solution. $50 \mathrm{mg}$ graphene oxide was mixed to $50 \mathrm{~mL}$ of anthocyanins extract and stirred continuously for $2 \mathrm{~h}$, the graphene oxide was dispersed uniformly mixed the extract solution. $0.2 \mathrm{Ml}$ of diluted $\mathrm{NH}_{3}$ solution was mixed above solution, the color was changed from red to green color. The reaction placed on the oil bath at $95{ }^{\circ} \mathrm{C}$ for $6 \mathrm{~h}$ and $12 \mathrm{~h}$ using water condenser to control the evaporation of solution. After complete the heat treatment, the reduced graphene oxide were obtained, filtered, washed with water and dried at $90{ }^{\circ} \mathrm{C}$ using hot air oven [10].

\section{Result and Discussion}

\section{A. Optical studies}

The band gap of the graphene oxide and reduced graphene oxide were measured using K-M plot from fig.1b, we obtained the band gap of the synthesized GO and rGO were measured found to be be $3.61 \mathrm{eV}$ and $3.38 \mathrm{eV}$ are shown in fig. 1 .

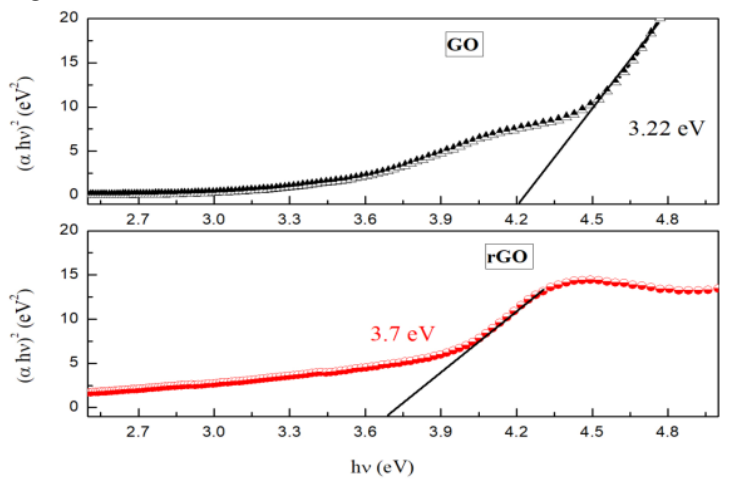

Fig.1 UV-band gap spectra

\section{B. Functional group analysis}

The peaks centered at 3424,3430 and $1625 \mathrm{~cm}-1$ can be assigned to the hydroxyl group, which reveals water linked to the products. The adsorption peaks at 1728 and $1053 \mathrm{~cm}-1$ can be attributed to the stretching of $\mathrm{C}=\mathrm{O}$ and $\mathrm{C}-\mathrm{O}$. The stretching vibrations of $\mathrm{C}-\mathrm{O}$ are shown at 1384 , respectively. The removal of such oxygen-containing groups of GO in all the rGO are clearly indicated by disappearance of the bands of $\mathrm{C}=\mathrm{O}$ stretching, $\mathrm{C}-\mathrm{O}-\mathrm{C}$ stretching, $\mathrm{C}-\mathrm{O}$ stretching and relative decrease in the intensity of broad band at $3400 \mathrm{~cm}^{-1}$ for the hydroxyl group [11] are shown in fig.2.

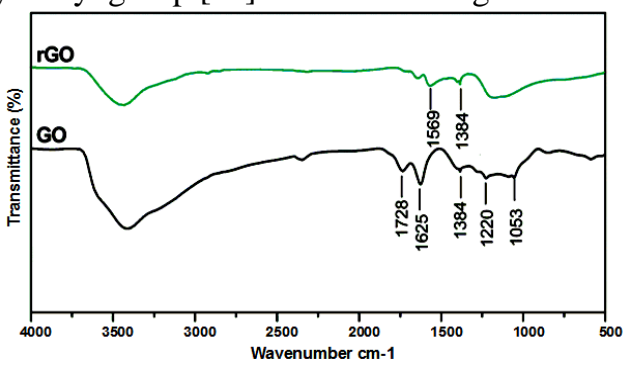

Fig. 2 FT-IR Spectra of GO and rGO
Crystalline structure analysis of GO and rGO are shown in fig.3. The peaks at $2 \theta$ are 23.85 and 43.04 , corresponding to planes (002) and (100) shows the presence of rGO with JCPDS card No.75-1621. The results confirm the conversion of $\mathrm{rGO}$ to reduced oxide by the chemical reduction by anthocyanins.

\section{Morphological structure analysis}

Morphological structure analysis is shown in fig. 4. These graphene oxide sheet contains more oxygen function groups in the surface on the graphene oxide, this oxygen function groups leads to the decreases the electrical conductivity.

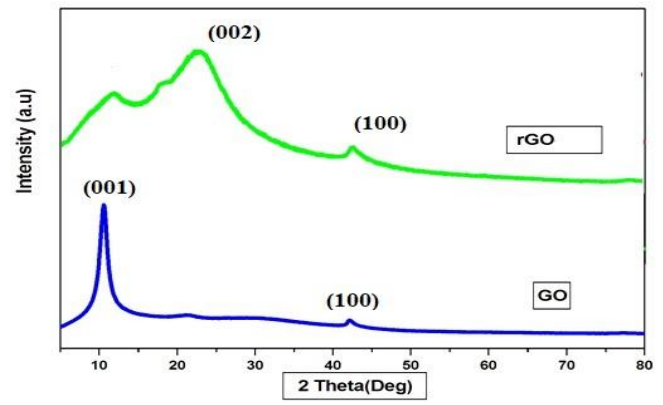

Fig. 3 XRD Spectra of GO and rGO

The graphene oxide sheets are thicker due to contains $\mathrm{C}=\mathrm{O}$ and $\mathrm{COOH}$ functional groups. The prepared graphene oxide treated with anthocyanins extract to form reduced graphene oxide as shown in fig. 4.

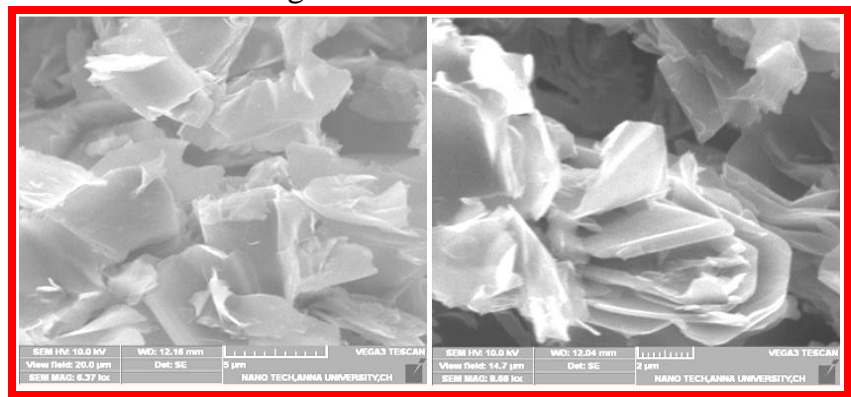

Fig.4 SEM analysis of GO and rGO

\section{E. Photocatalytic decomposition of MB}

The characteristic peak of MB is at $630 \mathrm{~nm}$. The UV absorption spectrum of reduced graphene oxide with $\mathrm{MB}$ almost removed up to 60 min under UV light irradiation as shown in fig.5a-b. The percentage of MB removal efficiency is to be measured. The decomposition of MB is shown in figure. The maximum efficiency $(85 \%)$ under UV light irradiation in $60 \mathrm{~min}$, the absence of catalyst, the MB intensity is gradually decreased by $4 \%$ due to the adsorption of the $\mathrm{MB}$ on the reduced graphene oxide in the dark condition. Decomposition efficiency of reduced graphene oxide under the same experimental condition are found to be $60 \%$ and $85 \%$ [12] are shown in fig.5a-b.

Different weight ratio of rGO towards decomposition of $\mathrm{MB}$ have been analysed various weight ratio of $\mathrm{rGO}$. The catalyst weight increase in $10 \mathrm{mg}$ to $30 \mathrm{mg}$ the $\mathrm{MB}$ decomposition ncreases. The reduced graphene oxide increases up to $30 \mathrm{mg}$ decreases the MB degradation performance as shown in

\section{Crystalline structure analysis}



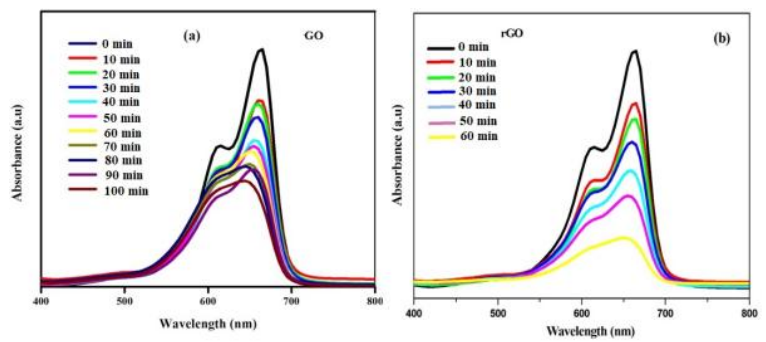

Fig.5 UV spectra of GO and rGO

fig. $7 \mathrm{~b}$. The optimum ratio of catalyst is $20 \mathrm{mg}$ for effective degradation of MB molecule [13].

\section{Conclusion}

As reduced rGO catalyst is effective degradation $\mathrm{MB}$ under UV light irradiation are reported. The XRD pattern of reduced graphene oxide confirms the presence of the reduced graphene oxide in crystalline nature. The SEM image of rGO shows that the thin sheet like structure after treated with anthocyanin. The functional identification from FT-IR bands at $1728,1053 \mathrm{~cm}^{-1}$ is to be ascribed $\mathrm{C}=\mathrm{O}$ and $\mathrm{C}-\mathrm{O}$ groups. The degradation efficiency of rGO is effective degradation when compare to GO.

\section{REFERENCES}

[1] J. P. A. de Mendonça, A. H. Lima, J. C. Roldao, J.S. Martins, G.M.A. Junqueira, W. G. Quirino, Fernando Sato, Mater. Chem. Phys. 215, (2018) 203-210.

[2] T. Lin, J. Chen, H. Bi, D. Wan, F. Huang, X. Xie, M. Jiang, J. Mater. Chem. 1 (3), (2013) 500-504

[3] X. Xua, X. Dinga, X. Yanga, P. Wanga, S. Lia, Z. Lua, H. Chena, J. Hazard. Mater. 364, (2019) 691-699.

[4] Y. Liu, X. Wu, Y. Tian, X. Zhou, B. Yu, Q. Zhang, R. Du, Q. Fu, F. Chen, Carbon 146, (2019) 618-626.

[5] G.G. Gebreegziabher, A.S. Asemahegne, D.W. Ayele, M. Dhakshnamoorthy, A. Kumar. Mater. Today Chem. 12, (2019) 233-239.

[6] A. Raja, P. Rajasekaran, K. Selvakumar, M. Arunpandian, K. Kaviyarasu, S. Asath Bahadur, M. Swaminathan, Separation and Purification Technology 233 (2020) 115996

[7] P. N. Khanam, A. Hasan, Int. J. Biol. Macromol. 126, (2019) 151-158.

[8] H. Khojasteh, H. Safajou, S. Mortazavi-Derazkola, M. Salavati-Niasari, K. Heydaryan, M. Yazdani, Journal of Cleaner Production. 229, (2019) 1139-1147.

[9] A. Raja, S. Ashokkumar, R. Pavithra Marthandam, J. Jayachandiran, Chandra Prasad Kathiwada, K. Kaviyarasu, R. Ganapathi Raman, M. Swaminathan, J. Photochem. Photobiol B. 181, (2018) 53-58.

[10] A. Raja, K. Selvakumar, P. Rajasekaran, M. Arunpandian, S. Ashokkumar, K. Kaviyarasu, S. Asath Bahadur, M. Swaminathan, J. Colloid Interface Sci. 564 (2019) 23-30.

[11] A. Raja, P. Rajasekaran, K. Selvakumar, M. Arunpandian, S. Asath Bahadur, M. Swaminathan, Electrochim. Acta. 328 (2019) 135062

[12] Sheng-Yaw Hsu, Sheng-Chi Lin, Jeng-An Wang, Chi-Chang Hu, Chen-Chi M. Ma, De-Hao Tsai, Electrochim. Acta. 296, (2019) 427-437.

[13] A. Raja, P. Rajasekaran, K. Selvakumar, M. Arivanandhan, S. Asath Bahadur, M. Swaminathan, Optik - International Journal for Light and Electron Optics 190 (2019) 21-27.

\section{AUTHORS PROFILE}

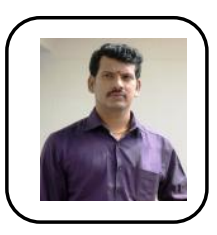

Dr. A. Raja is a Postdoctoral Fellow at Nanomaterials Laboratory, Department of Physics, International Research Centre, Kalasalingam Academy of Research and Education (Deemed to be University), Krishnankoil, India.

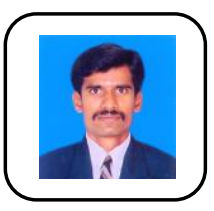

Dr. K. Selvakumar is a Postdoctoral Fellow at Nanomaterials Laboratory, Department of Chemistry, International Research Centre, Kalasalingam Academy of Research and Education (Deemed to be University), Krishnankoil, India. Now, he is working on the Postdoctoral Researcher, Institute of Microstructure and Property of Advanced Materials, Beijing University of Technology, Ping Le Yuan, Chaoyang, Beijing, China. He has published 21 papers in peer-reviewed journal on the kinetics, organic synthesis and photocatalysis.

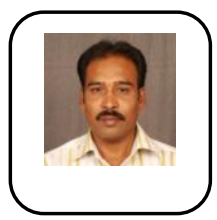

Dr. S. Asath bahadur is Professor in Department of Physics, International Research Centre, Kalasalingam Academy of Research and Education (Deemed to be University), Krishnankoil, India. His area of research is crystal growth, and Nanomaterials, Photocatalysis.

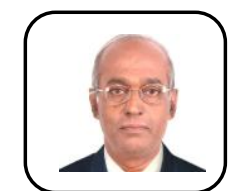

Dr. M. Swaminathan is Professor in Department of Chemistry, International Research Centre Kalasalingam Academy of Research and Education (Deemed to be University), Krishnankoil, India. His area of Research is photo chemistry. 